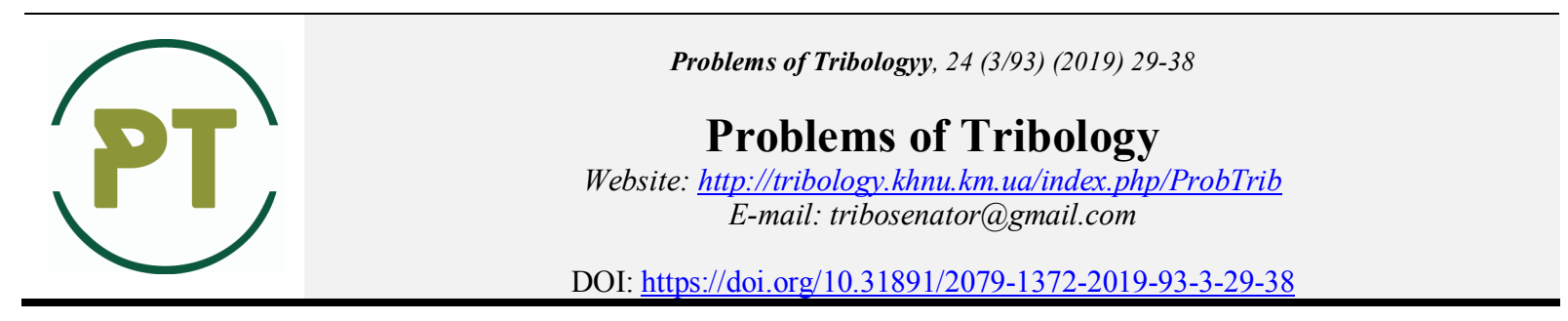

\title{
Estimation of the Stress-Strain State of a Discrete Medium by a Plastic Flow Model
}

\author{
O.A. Dorofeyev", V.V. Kovtun \\ Khmelnytskyi National University, Ukraine \\ *E-mail: $\underline{\text { sa dor@ukr.net }}$
}

\begin{abstract}
The article considers the possibility of using the apparatus of the plastic flow theory to evaluate the stress-strain state of a medium filled with discrete material. The ratio of the rheological model associated with the Coulomb condition is formulated, which takes into account the characteristic features of the deformation of discrete materials: the effect of dry coulomb friction and the manifestation of dilatation.

The ratios which describe the boundary condition of the plastic medium, both under the conditions of Saint-Venant and Mohr - Coulomb, are given. In the solid-state plastic flow model, the Saint-Venant condition is used as a potential function. Therefore, it is logical to consider the use of the Mohr - Coulomb condition as a potential function in the plastic flow model of a bulk medium. This is done in the Drucker - Prager model. The authors analyzed the validity of the findings of this model with respect to the discrete environment.

The ratio for the analysis of the reasons for the discrepancy between the conclusions of the Drucker Prager model and the results of experiments with discrete materials is given. The comparison of the magnitude of the dilatation coefficient with the condition of coincidence of the stress and deformed state in the limiting stage of deformation of the discrete medium, suggests that the Drucker - Prager model which is associated with the Coulomb condition doubles the value of this coefficient. The ratio of the Drucker - Prager model is related to the stresses acting on non-orthogonal planes of the boundary equilibrium, with deformations along the orthogonal planes of maximum displacement. This leads to a discrepancy between the predicted model and the experimentally determined values of the dilatation coefficient, as well as the impossibility to reconcile the fields of the slip lines in the stress and deformation plane.

The authors conclude that the highlighted differences between the theoretical conclusions and the results of experimental checks can be eliminated by presenting the relations of the model of the plastic flow of the discrete medium through the stresses and deformations that arise in the twisted conjugate sliding planes.
\end{abstract}

Key words: discrete environment; internal coulomb friction; dilatation.

Discrete is an environment filled with physically-discrete material: sand; rubble; granular, granular and similar natural or artificially created materials.

\section{Highlighting previously unresolved parts of a common problem}

An important task of engineering mechanics is to describe the contact interaction of such an environment with structural elements - with the foundations of structures, retaining walls, vehicles, mechanisms of technological processing of discrete materials. The complexity of solving these problems is the need for a joint description of the stress-strain state of structural elements and a discrete environment whose laws of deformation are much more complex than a rigid deformable body and which have not yet been sufficiently studied.

\section{Setting objectives}

Special experimental studies have found that the laws of deformation of discrete materials, as opposed to solids, must additionally reflect the following features: 
- influence of internal friction;

- manifestation of dilatancy (changes in material density during displacements);

- a significant dependence of the nature of the nonlinear physical dependences of "stress-strain" on the type of stress state (in the following we consider mainly plane-deformed state).

The purpose of the article is to evaluate the reliability of describing the stress-strain state of a discrete medium associated with a plastic flow model associated with the Coulomb boundary condition.

\section{Basic material and results}

The analysis of the rheological models that were proposed to describe the stress-strain state of the discrete medium [1] leads to the conclusion that the most complete features of deformation of this medium are capable of describing plastic flow models.

Unlike the "classical" models of the mechanics of a rigid deformable body, the determining relations of such models are not formulated as physical dependences between stresses $\{\sigma\}$ and deformations $\{\varepsilon\}$, but as a relationship between stresses and the rate of increase of plastic (residual) deformations $\{d \varepsilon\}$ [2]. This dependence is written in differential form

$$
\{d \varepsilon\}=d \lambda \frac{\partial \Phi(\{\sigma\})}{\partial\{\sigma\}}
$$

where $\Phi(\{\sigma\})$ - potential voltage function, or load function;

$d \lambda$ - the Lagrange multiplier.

Plastic deformation of materials is associated with the fulfillment of the condition of plasticity. If in the relation (1) for a potential function $\Phi(\{\sigma\})$ we accept the condition of plasticity, the ratio corresponds to the associated rheological model, in other cases, to an unassociated model.

\section{Model of plastic flow associated with the condition of plasticity of a solid}

In the theory of plasticity of a rigid body, the flat task is most often considered. The results of the experimental studies suggest that the flat deformation of plastic material is best described by the Saint-Venant condition:

$$
S=\tau_{\max }=0,5\left(\sigma_{1}-\sigma_{2}\right)=0,5 \sqrt{\left(\sigma_{x}-\sigma_{y}\right)^{2}+4 \tau_{x y}^{2}}=\tau_{\mathrm{T}},
$$

where $S=\tau_{\max } \Phi(\{\sigma\})$ - maximum tangential stress;

$\sigma_{1}, \sigma_{2}-$ the main stresses in the deformation plane;

$\sigma_{x}, \sigma_{y}, \tau_{x y}-$ normal and tangential stresses in the deformation plane $x y$;

$\tau_{\mathrm{T}}$ - material yield strength.

Under this condition, plastic deformation of the material occurs if the maximum shear stress $\tau_{\max }$ becomes equal to the yield point of the material $\tau_{T}$. Maximum tangential stresses $\tau_{\max }$ act on orthogonal shear planes that are inclined to the main angles $\pm \pi / 4$. It is between these planes that the maximum shear deformations occur $\gamma_{\max }$. Therefore, dependence (1) in this case describes the relationship between stresses $\{\sigma\}$ and strain rates $\{d \varepsilon\}$, occurring on the same planes, and can be considered as a determining relation of the plastic flow model associated with the Saint-Venant plasticity condition.

There are many stress boundary states that meet the Saint-Venan condition. These stress states can graphically be represented by a family of Mor boundary circles having a common envelope, parallel to the axis $\sigma$, which limits the zone of plastic deformation (Fig. 1).

Each of the boundary circles fully describes the stress state at one point of the plastic region through invariants $\sigma_{0}$ and $\tau_{\max }=\tau_{T}$.

Fig. 2 depicts one of the boundary circles of Mohr. The abscissa of the center $\mathrm{C}$ of the circle is equal to the average normal stress $\sigma_{0}=0,5\left(\sigma_{x}+\sigma_{y}\right)$, circle radius - maximum tangential stress $\tau_{\max }=\tau_{\mathrm{T}}$. The 
coordinates of the points $K$ and $K^{\prime}$ correspond to the stresses arising on the planes, inclined to the main angle $\alpha$. Points $A$ i $B$ - the main stresses $\sigma_{\min }, \sigma_{\max }$, and the points $M$ and $M^{\prime}$ with coordinates $\sigma_{0}=0,5\left(\sigma_{x}+\sigma_{y}\right)$, $\tau=\tau_{\max }=0,5\left(\sigma_{\max }-\sigma_{\min }\right)=\tau_{\mathrm{T}}-$ the scaffolds on which Saint-Venant condition is satisfied. These planes are inclined to the main angle $\pi / 4$ and in the model of rigid-plastic body are associated with sliding planes. The set of slip planes forms a grid of orthogonal slip lines (a grid of characteristics in the stress field). Normal $\sigma_{0}$ and tangents $\tau_{\mathrm{T}}$ stresses occur at each point of these lines.

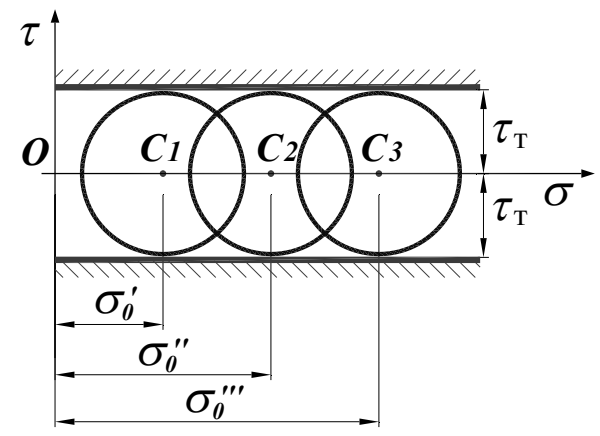

Fig. 1. Family boundary circles of Mohr

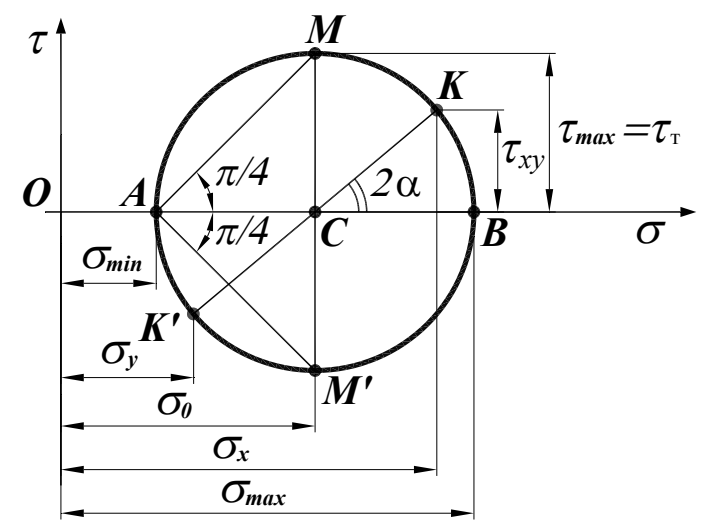

Fig. 2. Boundary circle of Mohr provided by Saint-Venant solid body.

Therefore, the grid of sliding lines clearly reflects the boundary stress state of the plane region of the

Fig. 3 shows an element of the flat region bounded by adjacent sliding lines. There are stresses on its

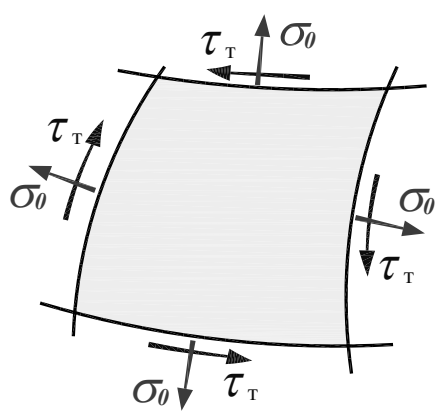

Fig. 3 The flat state of the element of the flat region

faces $\sigma_{0}=0,5\left(\sigma_{\max }+\sigma_{\min }\right)$ and $\tau_{\max }=0,5\left(\sigma_{\max }-\sigma_{\min }\right)=\tau_{\mathrm{T}}$, which are invariants of the plane stress state. This limit stress state can be represented as the sum of two simple ones: a comprehensive uniform stretch or a compression stress $\sigma_{0}$ and pure shear stress $\tau_{\mathrm{T}}$. On the principle of superposition, we obtain expressions for the principal stresses in the deformation plane $x y$ :

$$
\sigma_{\max }=\sigma_{0}+\tau_{\mathrm{T}} ; \sigma_{\min }=\sigma_{0}-\tau_{\mathrm{T}}
$$

as well as for stresses on arbitrary orthogonal planes inclined to the main angles $\alpha$ :

$$
\left.\begin{array}{l}
\sigma_{x}=\sigma_{0}+\tau_{\mathrm{T}} \cos 2 \alpha ; \\
\sigma_{y}=\sigma_{0}-\tau_{\mathrm{T}} \cos 2 \alpha ; \\
\tau_{x y}=-\tau_{y x}=\tau_{\mathrm{T}} \sin 2 \alpha .
\end{array}\right\}
$$

If the orientation of the plates with normals $x, y$ is determined relative to the sliding planes, it is sufficient to replace the angle in expressions (4) $\alpha$ by $\beta=\alpha+\pi / 4$. Relations (4) take the form

$$
\left.\begin{array}{l}
\sigma_{x}=\sigma_{0}+\tau_{\mathrm{T}} \sin 2 \beta ; \\
\sigma_{y}=\sigma_{0}-\tau_{\mathrm{T}} \sin 2 \beta ; \\
\tau_{x y}=-\tau_{y x}=-\tau_{\mathrm{T}} \cos 2 \beta .
\end{array}\right\}
$$

The dependences (4) make it possible to obtain a system of differential equations describing the characteristics of the field of boundary stresses (slip lines) as functions $\sigma_{0}$ and $\beta$.

The above correlations of the theory of plasticity cannot be directly used in the model of plastic flow of a discrete medium because the Saint-Venant criterion does not reflect the fundamental features of deformation of a discrete material in the limit state - the influence of internal friction and the manifestation of dilatancy. 


\section{Describing the limit state of a discrete medium}

The resistance to deformation of discrete materials is exerted by the forces of dry friction, which can occur only in the area of compressive stresses. Therefore, the criterion for the transition to the limit state of discrete materials, unlike plastic ones, must first and foremost reflect the influence of internal coulomb friction. Among such criteria, as shown by special studies [3], the boundary condition of the discrete medium for flat deformation conditions is most accurately described by the Mohr - Coulomb criterion.

By this criterion, the onset of the limit state of the discrete medium is determined not by the magnitude of the maximum tangent stresses $\tau_{\max }$, but by the magnitude of the maximum ratio of tangent and normal compressive stresses. The Mohr-Coulomb criterion can be written through the previously introduced flat stresses invariants with this expression

$$
\frac{\tau_{\max }}{\sigma_{0}}=\sin \varphi=\text { const }
$$

where $\varphi$ - angle of internal friction;

$\sigma_{0}$ - average compressive stress.

Therefore, the boundary state of a discrete material, at the occurrence of which plastic deformation of the environment occurs, is estimated by two parameters $\sigma_{0}$ and $\varphi$ and is graphically represented by a family of Morh boundary circles having a straight, curved axis that is inclined to the axis $\sigma$ at the angle of internal friction $\varphi$ (Fig. 4). All circles are characterized by the same ratio of parameters $R / \sigma_{0}=\sin \varphi$.

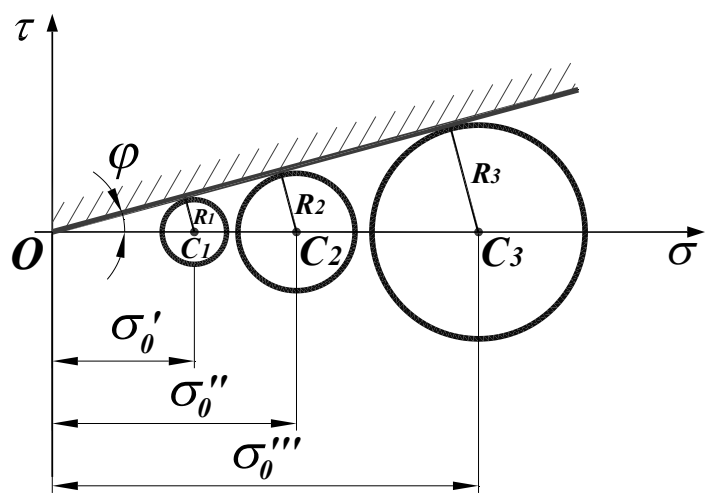

Fig. 4. A family of Mohr circles describing the limited state of the discrete environment

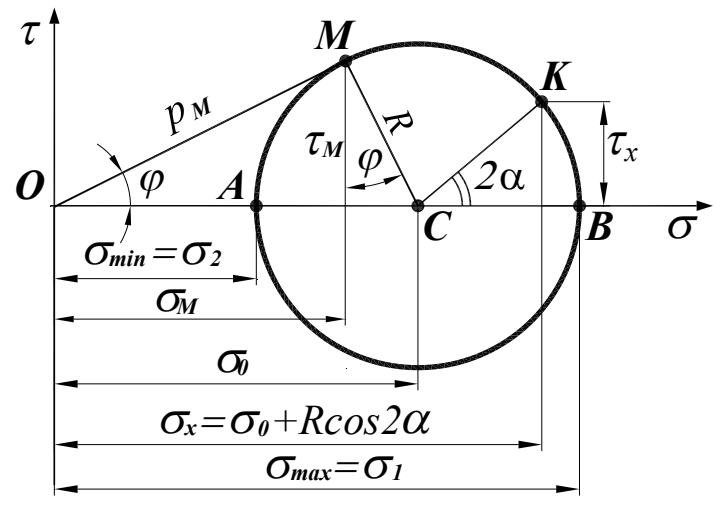

Fig. 5. The limited circle of stresses by the Mohr - Coulomb condition

The envelope line limits the area of the discrete material in which the boundary state occurs, and each of the circles describes the boundary stress state at the point of this region. One of the circles corresponding to the Morh - Coulomb criterion is shown in Fig. 5. Circle parameters are: radius $R=0,5\left(\sigma_{\max }-\sigma_{\min }\right)=0,5\left(\sigma_{1}-\sigma_{2}\right)$ and the abscissa of the center $C$ $\sigma_{0}=0,5\left(\sigma_{\max }+\sigma_{\min }\right)=0,5\left(\sigma_{1}+\sigma_{2}\right)$. A characteristic feature of a circle is that the tangent $O M$, drawn from the origin, is inclined to the axis $\sigma$ on the corner $\varphi$. Touch point $M\left(\sigma_{M}, \tau_{M}\right)$ corresponds to the plane with the maximum stress ratio $\frac{\tau_{M}}{\sigma_{M}}=\operatorname{tg} \varphi$, or platform with a maximum deviation of full stress $p_{M}$ from normal. Points $A$ and $B$ correspond to the principal stress planes $\sigma_{\min }=\sigma_{2}$ i $\sigma_{\max }=\sigma_{1}$, and all other points in the circle are arbitrary planes.

The Mohr circle (Fig. 5) graphically describes the boundary stress state at a point of a discrete medium by the Mohr - Coulomb criterion. Criterion (6) itself can be written by invariant parameter relations $R$ and $\sigma_{0}$ of a circle

$$
\frac{R}{\sigma_{0}}=\frac{\tau_{\max }}{\sigma_{0}}=\sin \varphi,
$$

due to the ratio of major compressive stresses $\sigma_{1}, \sigma_{2}$ : 


$$
\frac{R}{\sigma_{0}}=\frac{0,5\left(\sigma_{1}-\sigma_{2}\right)}{0,5\left(\sigma_{1}+\sigma_{2}\right)}=\frac{\sigma_{1}-\sigma_{2}}{\sigma_{1}+\sigma_{2}}=\sin \varphi,
$$

or because of the stress ratio $\tau_{M}, \sigma_{M}$ along the planes of boundary equilibrium (Fig. 5)

$$
\frac{\tau_{M}}{\sigma_{M}}=\operatorname{tg} \varphi
$$

Taken into account that the boundary state of a discrete Mohr - Coulomb criterion is associated with the magnitude of the stress ratios, it is sometimes convenient to represent it through a relative invariant $\xi=\sigma_{1} / \sigma_{2}$

$$
\frac{\sigma_{1}-\sigma_{2}}{\sigma_{1}+\sigma_{2}}=\frac{\xi-1}{\xi+1}=\sin \varphi .
$$

On the threshold of Mohr's circle (Fig. 5) directly we obtain expressions for major stresses $\sigma_{1}, \sigma_{2}$ through circle parameters

$$
\left.\begin{array}{l}
\sigma_{1}=\sigma_{0}+R=\sigma_{0}+\sigma_{0} \sin \varphi=\sigma_{0}(1+\sin \varphi) ; \\
\sigma_{2}=\sigma_{0}-R=\sigma_{0}-\sigma_{0} \sin \varphi=\sigma_{0}(1-\sin \varphi),
\end{array}\right\}
$$

or for stresses arising on an arbitrary plane with normal $r$ rotated about the principal axis 1 by an angle $\alpha$. These stresses correspond to the coordinates of point $K$ in Fig. 5

$$
\left.\begin{array}{l}
\sigma_{K}=\sigma_{0}+R \cos 2 \alpha=\sigma_{0}+\sigma_{0} \sin \varphi \cdot \cos 2 \alpha=\sigma_{0}(1+\sin \varphi \cdot \cos 2 \alpha) ; \\
\tau_{K}=R \sin 2 \alpha=\sigma_{0} \sin \varphi \cdot \sin 2 \alpha .
\end{array}\right\}
$$

To describe the boundary condition of a discrete medium, it is important to determine the stresses $\sigma_{M}$, $\tau_{M}$ on platforms with maximum deviation of total stress $p_{M}$ from normal. With Mohr's circle we get:

$$
\left.\begin{array}{l}
\sigma_{M}=\sigma_{0}-R \sin \varphi=\sigma_{0}-\sigma_{0} \sin ^{2} \varphi=\sigma_{0}\left(1-\sin ^{2} \varphi\right)=\sigma_{0} \cos ^{2} \varphi ; \\
\tau_{M}=R \cos \varphi=\sigma_{0} \sin \varphi \cdot \cos \varphi=0,5 \sigma_{0} \sin 2 \varphi .
\end{array}\right\}
$$

Platforms with maximum stress ratio $\tau_{M} / \sigma_{M}$ tilted to the main angles $\pi / 4 \pm \varphi / 2$ and in the statics of a free-flowing environment, they are associated with slip planes. The set of slip planes in the plane of deformation $x, y$ form a grid of non-orthogonal sliding lines that intersect at angles $(\pi / 2 \pm \varphi)$.

Fig. 6 shows two sliding lines intersecting at point A. The major axis 1 forms with the $x$-axis $\alpha$, and

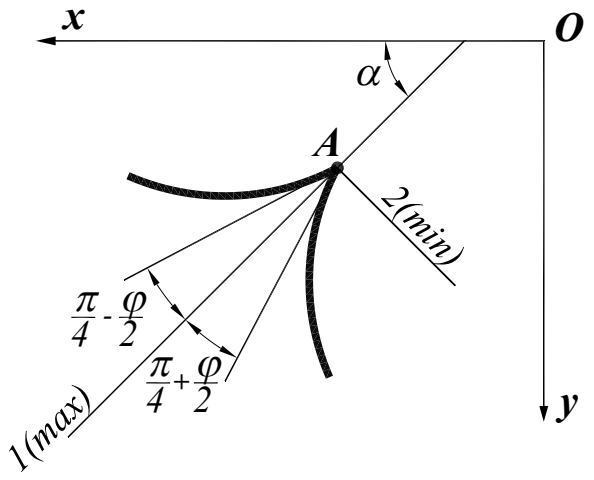

Fig. 6. Orientation of sliding lines tangent lines are angles $\alpha+(\pi / 4 \pm \varphi / 2)$. Since the MohrCoulomb condition determines the orientation of the slip planes, equations of slip lines (characteristic lines) in the stress field are directly obtained from expressions (12), (13). The system of these differential equations was studied in detail by V.V. Sokolovsky [4] and used by him to solve engineering problems of free-flow static.

Relations (3) - (5) describe the limit state of the plastic medium under the condition of Saint-Venant. Similar relations (6) - (13) are the boundary state of a discrete medium under the Mohr-Coulomb condition. In the solid-state plastic flow model, the Saint-Venan condition is used as a potential function $\Phi(\{\sigma\})$. Therefore, it is logical to consider the use of the Mohr-Coulomb condition as a potential function in the plastic flow model of a bulk medium. This model was first proposed by D. Drucker and W. Prager [5].

\section{The ratio of the rheological model associated with the Coulomb plastic flow model}

Potential function $\Phi(\{\sigma\})$ of the Drucker - Prager model, according to the fundamental relation (1), relates the strain rate $\{d \varepsilon\}$ with limit stresses $\{\sigma\}$.

The choice of the potential function of the model in the form of the Coulomb condition (6) allows to determine the direction of the strain velocity vector $\{d \varepsilon\}$ in the system of orthogonal axes $d \varepsilon_{0}-d \gamma_{\max }$. 
Indeed, from the ratio (1) $\{d \varepsilon\}=d \lambda \frac{\partial \Phi(\{\sigma\})}{\partial\{\sigma\}}$ the components of the vector are directly determined $\{d \varepsilon\}\left(d \varepsilon_{0}, d \gamma_{\max }\right)$ due to stress $\sigma_{0}, \tau_{\max }:$

$$
\left.\begin{array}{l}
d \varepsilon_{0}=d \lambda \frac{\partial \Phi}{\partial \sigma_{0}}=-d \lambda \frac{\tau_{\max }}{\sigma_{0}^{2}} \\
d \gamma_{\max }=d \lambda \frac{\partial \Phi}{\partial \tau_{\max }}=d \lambda \frac{1}{\sigma_{0}}
\end{array}\right\}
$$

and their relationship

$$
\Delta=\frac{d \varepsilon_{0}}{d \gamma_{\max }}=-\frac{\tau_{\max }}{\sigma_{0}}=-\sin \varphi
$$

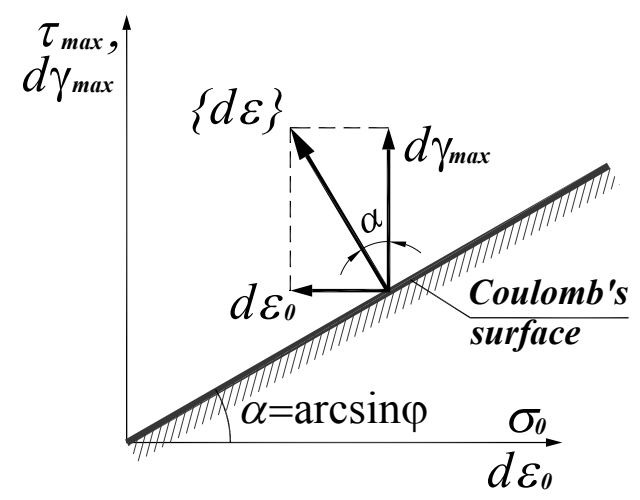

Fig. 7. The combination of stresses and strain rates

Value $\Delta$, which relates the ratio of displacement rates to displacement $d \varepsilon_{0} / d \gamma_{\max }$ with boundary stress ratio $\tau_{\max } / \sigma_{0}$, can be seen as a measure of dilation. It was called the dilatancy ratio.

According to expression (15), the strain velocity vector $\{d \varepsilon\}$ in the coordinate system $d \varepsilon_{0}-d \gamma_{\max }$ should be perpendicular to the Coulomb boundary surface, which is clearly shown in the fig. 7, which combines systems of orthogonal coordinates of boundary stresses $\left(\sigma_{0}-\tau_{\max }\right)$ and strain rates $\left(d \varepsilon_{0}-d \gamma_{\max }\right)$. Coulomb boundary surface in the axis system $\sigma_{0}-\tau_{\max }$ is described as straight $\sigma_{0}=k \tau_{\max }$ with an angular coefficient $k=\operatorname{tg} \alpha, \alpha=\arcsin \varphi$.

The perpendicularity of the vector $\{d \varepsilon\}$ to the boundary surface is an important conclusion associated with the Coulomb condition (6) of the Drucker - Prager model, which requires special experimental and theoretical verification with respect to discrete materials.

The use of the Mohr-Coulomb condition as a potential function in the Drucker-Prager model not only takes into account the influence of internal friction and the manifestation of dilatation on the deformation process of a discrete material, but also establishes a relation between the field of boundary stresses and the field of strain rates in the array of discrete medium. Laboratory verification of dependence (15) has shown that the theoretical value of the dilatation coefficient $\Delta$ in most cases significantly exceeds the set experimentally.

For example, the angle of internal friction $\varphi$ dry sand is about $30^{\circ}\left(\Delta=\sin 30^{\circ}=0,5\right)$. In the experiments of A.S. Stroganov the coefficient of dilatation of dense sand was $\Delta=0,239 \quad\left(\sin 14^{\circ}\right)$, in experiments by P. Roscoe and S. Friedman the value $\Delta$ was changed from $\Delta=0,1$ to $\Delta=0,35$. Not only the fluffing but also the compaction of loose sands was noted.

More thoroughly, the reliability of describing the stress-strain state of a discrete material by the Drucker - Prager model can be investigated by comparing the orientation of the planes on which the Mohr Coulomb condition in the stress field is realized, with the orientation of the slip planes in the field of strain rates.

In classical plasticity theory, the Saint-Venant condition is realized on orthogonal planes inclined to the principal angles $\pm \pi / 4$. On these orthogonal planes, maximum shear deformations occur $d \gamma_{\max }$. Therefore, 
with the coincidence of the directions of the principal axes of the stress tensor and the strain rate tensor, the stress fields and strain rates coincide.

In the model of Drucker - Prager orientations of non-orthogonal planes of boundary equilibrium and sliding planes with maximum shear deformation rates $d \gamma_{\max }$ cannot coincide in reality, since the grid of nonorthogonal sliding lines in the field of boundary stresses and the grid of orthogonal sliding lines in the deformation field cannot be reconciled with linear transformations of coordinate systems. The specified coincidence of slip lines in the field of boundary stresses and strain rates can be explained by incorrect use of the apparatus of the theory of plasticity of the solid body to describe the stress-strain state of the discrete material.

The theory of plasticity of a rigid body operates with dependences between stresses and strains arising on orthogonal planes of maximum shear $\gamma_{\max }$. This agrees with the description of the stress state in the condition of Saint-Venant with a deformed state.

The relation of the same Drucker - Prager model combines the dependences between the stresses acting on non-orthogonal planes of boundary equilibrium - on the planes with the maximum stress ratio $\tau_{M} / \sigma_{M}=\operatorname{tg} \varphi$, with dependencies between strain rates $d \varepsilon_{0}, d \gamma_{\max }$ on orthogonal planes of maximum displacement. This mismatch of stress and strain fields can be eliminated if, in the determining relation of the Drucker-Prager model, the deformed state of the medium is represented by the strain rates $d \varepsilon_{M}, d \gamma_{M}$ on nonorthogonal planes of boundary equilibrium, not on planes of maximum displacement $d \varepsilon_{0}-d \gamma_{\max }$.

Since the theory of plasticity of a rigid body does not consider deformations on non-orthogonal planes, it is proposed to describe the boundary stress and corresponding deformed state of the discrete medium due to stresses and rates of deformations arising on special conjugate planes - in even planes with equal magnitude $\tau / \sigma=\operatorname{tg} \eta$.

The description of the stress state due to the stress on the coupled planes is discussed in article [6], and the analysis of the deformed state is discussed in article [7].

The class of conjugate planes includes the planes of boundary equilibrium for which $\tau_{\mu} / \sigma_{M}=\operatorname{tg} \eta_{\max }=\operatorname{tg} \varphi$. These planes were used by S.S. Golushkevich [8] and P.I. Yakovlev [9] to develop effective engineering deformation-free methods for estimating the boundary stress state of the soil environment.

To determine shear deformations $\gamma$ between non-orthogonal directions it is proposed [7] to represent the deformed state of the medium due to linear deformations $\varepsilon_{r}$ and rotation (shift) $\vartheta_{r}$ arbitrary direction $r$ relative to the principal axis 1 .

Shift $\vartheta_{r}$ in the direction $r$, which is inclined to the principal axis by an angle $\alpha$, is determined by major deformations $\varepsilon_{1}, \varepsilon_{2}$ addiction

$$
\vartheta_{r}=0,5\left(\varepsilon_{1}-\varepsilon_{2}\right) \sin 2 \alpha,
$$

the use of which allows to determine the displacement of the displacement $\gamma_{a b}$ between any non-orthogonal directions $a$ and $b$, inclined to the main axis at angles $\alpha$ and $\beta$,

$$
\gamma_{a b}=\vartheta_{a}+\vartheta_{b}=0,5\left(\varepsilon_{1}-\varepsilon_{2}\right)(\sin 2 \alpha+\sin 2 \beta) .
$$

As shown in [7], the dependencies between linear $d \varepsilon_{r}$ and angular $d \vartheta_{r}$ deformations as opposed to dependencies $d \varepsilon-d \gamma$, used in the theory of rigidity plasticity, similar to the dependence between stresses $\sigma-\tau$. That is, circle diagrams that illustrate the tense $(\sigma-\tau)$ and deformed $(d \varepsilon-d \vartheta)$ the states are similar.

To use the same relationships between stresses and strains on the conjugate planes in the plastic flow model of a discrete material, it is necessary to provide not only the similarity of circular diagrams of boundary stresses $(\sigma-\tau)$ and strain rates $(d \varepsilon-d \vartheta)$, and their full coincidence.

Fig. 8 shows a circular diagram (Mohr circle) describing the variation of the stresses $\sigma, \tau$ at the point of the discrete medium at the rotation of the normal of the stress plane by the angle $\alpha$. Points $M$ and $M_{1}$ with coordinates $\sigma_{M}, \tau_{M}$ correspond to paired conjugate planes of boundary equilibrium - planes of the greatest deviation of total stress $p_{M}$ from normal.

Deformation dependencies describing the change in deformation $d \varepsilon_{r}, d \vartheta_{r}$ in the limiting state of the discrete material at normal rotation $r$ the deformation plane by the angle $\beta$ clearly shows the circle diagram $d \vartheta_{r}-d \varepsilon_{r}$ (Fig. 9). 
The diagram will completely coincide with the boundary circle of Mohr (Fig. 8), if tangent $\mathrm{Om}$, drawn to it from the origin will be tilted to the axis $d \varepsilon_{r}$ at the angle of internal friction $\varphi$. In this case, the coordinates of the touch point $m$ will be deformed $d \varepsilon_{r m}, d \vartheta_{r m}$ normal direction $r$ plane of the boundary equilibrium for which the ratio of deformations $d \vartheta_{r m} / d \varepsilon_{r m}=\operatorname{tg} \varphi$.

Therefore, the condition of coincidence of the boundary stress and deformed states of a discrete medium can be formulated as an equality of stress relations $\tau_{\mu} / \sigma_{M}$ and strain relations $d \vartheta_{r m} / d \varepsilon_{r m}$ perpendicular to the sliding plane of the direction:

$$
\frac{d \vartheta_{r m}}{d \varepsilon_{r m}}=\frac{\tau_{M}}{\sigma_{M}}=\operatorname{tg} \varphi
$$

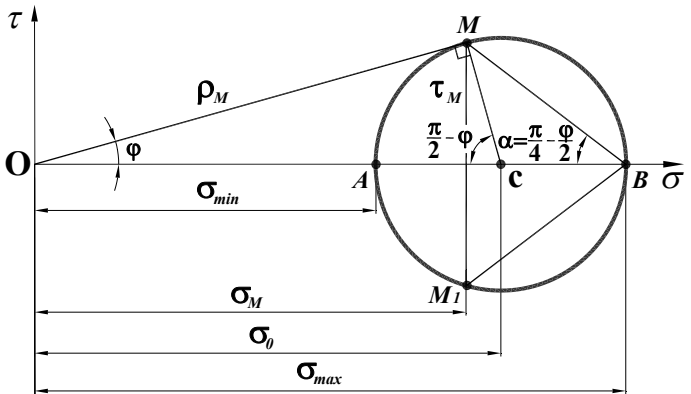

Fig. 8. Mohr's boundary circle

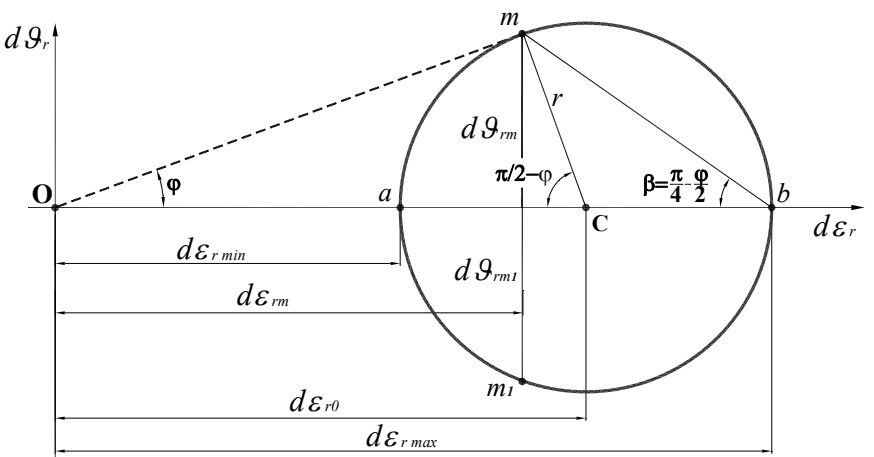

Fig. 9. Circle diagram $d \vartheta_{\mathrm{r}}-d \varepsilon_{\mathrm{r}}$ of strain rates of discrete environment in the limited state

Condition (18) can be used to analyze the reliability of the description of the Drucker-Prager model of the stress-strain state of the discrete medium, given that the relations of the model use deformations occurring in the directions of the deformation planes and not in the directions of their normals. Let us represent the condition of coincidence of the boundary stress and deformed states due to deformations $d \varepsilon_{m}, d \vartheta_{m}$ on the sliding surfaces by rotating the coordinate axes $d \varepsilon_{r}, d \vartheta_{r}$ (Fig. 9) on $90^{\circ}$. In this case, the matching condition is determined by the dependency^

$$
\frac{d \vartheta_{m}}{d \varepsilon_{m}}=-\frac{\tau_{M}}{\sigma_{M}}=-\operatorname{ctg} \varphi, \text { або } \frac{d \varepsilon_{m}}{d \vartheta_{m}}=-\frac{\sigma_{M}}{\tau_{M}}=-\operatorname{tg} \varphi,
$$

and shear deformation $d \gamma_{m m_{1}}$ between paired conjugate sliding planes $m, m_{1}$ is determined from (17). Taking into account that $d \vartheta_{m}=d \vartheta_{m_{1}}$, and $\alpha_{m}=\beta_{m_{1}}=\pi / 4+\varphi / 2$, we get:

$$
d \gamma_{m m_{1}}=\left(d \varepsilon_{1}-d \varepsilon_{2}\right) \sin (\pi / 2+\varphi) \text {. }
$$

We use the above relations to analyze the reasons for the discrepancy between the findings of the Drucker - Prager model and the results of experiments with discrete materials.

Let us introduce a potential function $\Phi(\{\sigma\})$ associated with the Mohr-Coulomb condition of a plastic flow model of a discrete material due to the stress ratio $\tau_{M} / \sigma_{M}$ on the sliding surfaces in the form (9):

$$
\Phi(\{\sigma\})=\frac{\tau_{M}}{\sigma_{\mathcal{M}}}=\operatorname{tg} \varphi .
$$
deformation

From the fundamental relation (1) of the theory of plastic flow, we obtain expressions for the rates of

$$
\left.\begin{array}{l}
d \varepsilon_{m}=d \lambda \frac{\partial \Phi(\{\sigma\})}{\partial \sigma_{\mu}}=-d \lambda \frac{\tau_{\mu}}{\sigma_{\mu}^{2}} ; \\
d \gamma_{m}=d \lambda \frac{\partial \Phi(\{\sigma\})}{\partial \tau_{\mu}}=d \lambda \frac{1}{\sigma_{\mu}} ;
\end{array}\right\}
$$




$$
\Delta_{m}=\frac{d \varepsilon_{m}}{d \gamma_{m}}=-\frac{\tau_{M}}{\sigma_{M}}=-\operatorname{tg} \varphi,
$$

which are similar to the relations (14), (15) recorded in the stresses $\sigma_{0}, \tau_{\max }$.

The dependence (23) reflects the condition of the perpendicularity of the vector $\{d \varepsilon\}\left(d \varepsilon_{m}, d \gamma_{m}\right)$ to the Coulomb boundary surface presented in formula (9) or the condition of the perpendicularity of the slip lines in the field of boundary stresses $\{\sigma\}\left(\sigma_{\mu}, \tau_{\mu}\right)$ and the deformation field $\{d \varepsilon\}\left(d \varepsilon_{m}, d \gamma_{m}\right)$.

This conclusion is inconsistent with the condition of coincidence of the boundary stress and strain states (19), which is formulated as the equality of stress relations $\tau_{\mu} / \sigma_{M}$ and deformations $d \varepsilon_{m} / d \vartheta_{m}$, not the strain ratio $d \varepsilon_{m} / d \gamma_{m}$.

To evaluate the validity of the conclusions of the Drucker - Prager model with respect to discrete material, we present the dilatation coefficient $\Delta_{m}$ (23) due to shear deformation $\vartheta_{m}$ the plane of boundary equilibrium and the main plane, not due to shear deformation $\gamma_{m}$ between paired sliding planes. Taking into account that $d \gamma_{m}=2 \vartheta_{m}$, from expression (23) we obtain:

$$
\Delta_{m}=\frac{d \varepsilon_{m}}{2 \vartheta_{m}}=-\frac{\tau_{M}}{\sigma_{m}}=-\operatorname{tg} \varphi, \text { або } \frac{d \varepsilon_{m}}{d \vartheta_{m}}=-2 \operatorname{tg} \varphi .
$$

Comparing the dilatancy coefficient $\Delta_{m}$ (24) with condition (19) of the coincidence of the stress and deformed state in the limit stage of deformation of the discrete medium one can claim that the Drucker - Prager model, associated with the Coulomb condition, is twice bigger than the value of the coefficient $\Delta_{m}$. This explains the discrepancies between the theoretical data and the results of the experiments described above.

\section{Conclusions}

The results of the research presented in the article allow us to draw the following conclusions:

1) rheological model of Drucker - Prager, unlike models of plastic flow of solid body, takes into account the fundamental features of the laws of deformation of discrete materials - the influence of internal friction and the manifestation of dilatancy;

2) the relation associated with the Coulomb condition of the Drucker - Prager model is related to stresses appearing on non-orthogonal planes of boundary equilibrium with deformations on orthogonal planes of maximum displacement. This leads to a discrepancy between the predicted model and experimentally determined values of the dilatancy coefficient, as well as the inability to reconcile the fields of the slip lines in the planes of stresses and strain rates.;

3) the marked differences between the theoretical conclusions and the results of the experimental tests can be eliminated by presenting the model relationships of the plastic flow of the discrete medium due to stresses and deformations arising on the paired conjugate sliding planes.

\section{References}

1. Dorofeyev O.A. Reolohichni modeli seredovyshcha $\mathrm{z}$ suttievym vnutrishnim tertiam / O.A. Dorofeyev, V.V. Kovtun // Visnyk KhNU. - Tekhnichni nauky. - 2019. - № 3 (271). - S. 50-58.

2. Sedov L. I. Mehanika sploshnoy sredyi / L. I. Sedov. - M. : Nauka, 1970. - T. $2-578$ s.

3. Kovtun V.V. Yssledovanye prochnosty sypuchykh materyalov v uslovyiakh ploskoi deformatsyy / V.V. Kovtun, E.V. Bahryi, V.T. Buhaev // Budivelni konstruktsii. - 2004. - Vyp. 61. - t. 1. - S. 109-116.

4. Sokolovskiy V.V. Statika syipuchey sredyi / V.V. Sokolovskiy. - M. : Nauka, 1960. - $272 \mathrm{~s}$.

5. Drucker D.C. Solid Mechanics and Plastic Analysis of Limit Design / D.C. Drucker, W. Prager. // Quarterly of Applied Mathematics. - 1952. - Vol. 10. - №2. - P. 157-165.

6. Kovtun .V. Napruzhennia po potentsialnykh ploshchynkakh kovzannia u sypkomu seredovyshchi / V.V. Kovtun // Visnyk KhNU. - Tekhnichni nauky. - 2010. - № 1. - S. 7-12.

7. Kovtun V.V. Deformatsii vzdovzh potentsialnykh linii kovzannia u sypkomu seredovyshchi / V.V. Kovtun, O.A. Dorofieiev. // Visnyk Khmelnytskoho natsionalnoho universytetu. Tekhnichni nauky. 2010. - №5. - S. 142-150.

8. Golushkevich S.S. Statika predelnyih sostoyaniy gruntovyih mass / S.S. Golushkevich. - M. : Gostehizdat, 1958. $-288 \mathrm{~s}$.

9. Yakovlev P.I. Predelnoe sostoyanie po S.S. Golushkevichu i tehnicheskaya teoriya predelnogo napryazhennogo sostoyaniya gruntovoy sredyi / P.I. Yakovlev. - Odessa : Astroprint, 2008. - 230 s. 
Дорофєєв О.А., Ковтун В.В. Оцінка напружено-деформівного стану дискретного середовища моделлю пластичного плину.

В статті розглядається можливість використання апарату теорії пластичного плину для оцінки напружено-деформованого стану заповненого дискретним матеріалом середовища. Формулюються співвідношення асоційованої 3 умовою Кулона реологічної моделі, які враховують характерні особливості деформування дискретних матеріалів: вплив сухого кулонового тертя і прояв дилатансії. Проаналізована достовірність висновків відомої моделі Друкера-Прагера щодо дискретного середовища.

Наведені співвідношення, що описують граничний стан пластичного середовища. Логічним слід вважати використання умови Мора - Кулона як потенціальної функції в моделі пластичного плину сипкого середовища, що зроблено в моделі Друкера-Прагера. Автори проаналізували достовірність висновків даної моделі щодо дискретного середовища.

Співвідношення моделі Друкера - Прагера пов'язують напруження, що діють по неортогональних площинках граничної рівноваги, з деформаціями по ортогональних площинках максимального зсуву. Саме це призводить до неспівпадіння передбачених моделлю і експериментально визначених величин коефіцієнта дилатансії, а також до неможливості узгодити поля ліній ковзання в площинах напружень і швидкостей деформацій.

Авторами зроблено висновок, що відмічені розбіжності між теоретичними висновками i результатами експериментальних перевірок можна ліквідувати шляхом представлення співвідношень моделі пластичного плину дискретного середовища через напруження і деформації, які виникають по парних спряжених площинках ковзання.

Ключові слова: дискретне середовище, внутрішнє кулонове тертя, дилатансія. 